\title{
Primeros datos sobre la distribución, tamaño poblacional y reproducción del picamaderos negro Dryocopus martius (Linnaeus, 1758) en un área del País Vasco de reciente colonización.
}

Preliminary data on the distribution, population size and reproduction of the black woodpecker Drycopus martius (Linnaeus, 1758) in a recentlycolonised area of the Basque Country.

Aitor Galarza ${ }^{1} 2^{*}$, Igor Aginako², Aitor Ballesteros ${ }^{2}$, Xabier Barreiro $^{2}$, Cristina Cinos ${ }^{2}$, Eneko Díaz ${ }^{2}$, Ander Egia², Juan Ramón Egia², Luki Fondado², Iñaki Garmendia², Antonio González ${ }^{3}$, Diego Hijosa ${ }^{3}$, Urko lbáñez ${ }^{2}$, Iñigo Iriarte² ${ }^{2}$ Francisco Martínez², Jesús Mari Molledo ${ }^{2}$, Iker Novoa², Juan Manuel Pérez de Ana², Julio Ruiz², Gorka Vacas², Santiago Vallejo ${ }^{3}$, Ernesto Vega ${ }^{2}$

$\xi^{*}$

\section{Resumen}

La distribución del picamaderos negro en la península lbérica ha experimentado recientemente una expansión que ha dado lugar a la coalescencia de sus poblaciones cantábrica y pirenaica. El presente estudio analiza la distribución de la especie y realiza una estima de su abundancia en una de las zonas de contacto recientemente colonizadas. Asimismo, este trabajo aporta información sobre sus preferencias ecológicas a nivel regional. Para ello se recopilaron las citas de los últimos años y se realizaron itinerarios de censo, así como una

\footnotetext{
1 Sociedad de Ciencias Aranzadi. Departamento de Ornitología Zorroagagaina 11, 20014 Donostia

2 Diputación Foral de Bizkaia. Departamento de Sostenibilidad y Medio Natural Alda. Rekalde, 30, 48009 Bilbao

3 Junta de Castilla y León. Oficina comarcal de Agentes Medioambientales Estación s/n, 09586 Nava de Ordunte, Burgos
}

* Correspondencia: aitorgalarzai@gmail.com 
búsqueda intensiva de nidos durante la primavera de 2019. También se analizó la tipología forestal en el entorno de los nidos, las características de los árboles-nido y la productividad media. Se registraron 375 citas repartidas por gran parte del área de estudio, estimándose un mínimo de 50 territorios. Se observó que la cobertura de pino, haya y roble americano en torno a los nidos fue mayor que la esperada mientras que fue menor la de eucalipto, encina y bosque de ribera. Como árbol-nido utilizaron ejemplares de haya, pino de Monterrey, pino marítimo y roble americano, emplazados en parcelas de estos tipos de arbolado. También utilizaron eucaliptos viejos que crecían en repoblaciones de coníferas. El éxito de cría fue elevado (80\%) pero la tasa media de pollos volados $(2,1)$ fue inferior a la media observada en otras regiones europeas. Se concluye que las coníferas están jugando un importante papel en la colonización y que su sustitución por cultivos de eucaliptos podría limitar el proceso expansivo.

Palabras clave: pájaros carpinteros, Bizkaia, colonización, hayedos, repoblaciones, Pinus radiata, Eucalyptus spp.

\begin{abstract}
The distribution area of the black woodpecker in the Iberian Peninsula has expanded in recent years, leading to a unification of its Cantabrian and Pyrenean populations. The present study evaluates the distribution of this species and estimates its abundance in one of the recently-colonised contact areas. Likewise, this work provides information on their ecological preferences at a regional level. To this end, records from the last years were compiled and line transects were carried out, as well as an intensive search for nests during the spring of 2019. We also analysed the forest type in the area surrounding the nests, the characteristics of the nest-trees and the average rate of chicks per nest. 375 records were compiled throughout much of the study area, estimating a minimum of 50 territories. It was observed that the coverage of pine, beech and American oak around the nests was greater than expected, while that of eucalyptus, holm oak and riverside forests was lower. The species of nest-trees they used were beech, Monterey pine, maritime pine and American oak, located in areas with the same types of trees. They also used old eucalyptus trees that grew in coniferous plantations. Breeding success was high (80\%) but the average rate of fledglings per nest (2.1) was lower than the average observed in other European regions. We conclude that conifers are playing an important role in colonisation and that their replacement by eucalyptus plantations may limit the expansion process.
\end{abstract}

Key words: woodpeckers, Biscay, colonisation, beech grove, plantations, Pinus radiata, Eucalyptus spp.

\title{
Laburpena
}

Okil beltzaren banaketak hedapen handia izan du Iberiar penintsulan berriki, eta horrek kantaurialdeko eta Pirinioetako populazioen bat-egitea eragin du. Ikerketa honek espeziearen banaketa aztertu eta populazioaren tamaina estimatu du. Era berean, lan honek 
eskualde mailan dituzten lehentasun ekologikoei buruzko informazioa ematen du. Horretarako, azken urteetako aipamenak bildu, trantsektuak egin eta habiak bilatzeko lan intentsiboa egin zen 2019ko udaberrian. Aztertu ziren, halaber, habiaren inguruko basoen tipologia, habietarako zuhaitzen ezaugarriak eta batez besteko txita tasa ere. 375 aipu jaso ziren azterketa eremuan zehar eta gutxienez 50 lurralde estimatu ziren. Ikusi zen habien inguruan pinuen, pagoen eta haritz amerikarren estaldura espero baino handiagoa zela, eta txikiagoa, aldiz, eukalipto, artadi eta ibar-basoena. Habietarako zuhaitz espezie hauek erabili zituzten: pagoa, intsinis pinua, itsas pinua eta haritz amerikarra, zuhaitz mota hauetako lursailetan kokatuak. Konifero landaketetan tartekatuta hazten ziren eukalipto zuhaitz zaharrak ere erabiltzen zituzten. Ugaltze arrakasta handia izan zen (\% 80), baina habia bakoitzean txita hegaldatuen batez besteko tasa $(2,1)$ Europako beste eskualde batzuetan ikusitakoa baino txikiagoa zen. Hortaz, ondorioztatu da koniferoek kolonizazioan funtzio garrantzitsua betetzen dutela eta pinudiak eukalipto landaketekin ordezkatzeak hedapen prozesua mugatu egin dezakeela.

Gako hitzak: okilak, Bizkaia, kolonizazioa, pagadiak, landaketak, Pinus radiata, Eucalyptus spp.

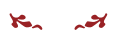

\section{Introducción}

Se considera al picamaderos negro Dryocopus martius (Linnaeus, 1758) una especie clave de los ecosistemas forestales porque proporciona cavidades que son utilizadas por otros vertebrados cuyas necesidades ecológicas se ven limitadas por las prácticas intensivas de la silvicultura moderna (Johnson et al., 1990; Rolstad et al., 2000; Mikusinski et al., 2001, Konsinski et al., 2010). Es un pájaro carpintero ampliamente distribuido por el Paleártico, desde el norte de la península Ibérica hasta Japón. Aunque tradicionalmente su presencia ha sido asociada a los bosques maduros (Del Hoyo et al., 2002), numerosos autores han enfatizado su capacidad para adaptarse a distintos ambientes forestales (Tjernberg et al., 1993, Rolstad et al., 1998; Mikusinski et al., 2001; Bocca et al., 2007; Pirovano \& Zecca, 2014).

Su área de distribución europea, que se extiende desde los bosques boreales escandinavos hasta los hayedos del sur de Italia, ha experimentado una importante ampliación desde finales del siglo XIX (Gorman, 2011). Más recientemente, también se ha observado la expansión de la población ibérica (Camprodon et al., 2007), lo que ha dado lugar a la fusión de sus dos núcleos poblacionales del norte de España (Gainzarain \& Fernández-García, 2013), el pirenaico, que en el siglo pasado se extendía desde Cataluña hasta la Sierra de Aralar, con el cantábrico, que alcanzaba desde la montaña occidental de Cantabria hasta la Sierra de Los Ancares, y que se encontraba aislado hasta entonces del resto de la población europea (Simal \& Herrero, 2003). 
Hasta finales del siglo pasado la constatación de su presencia en el País Vasco se limitaba a observaciones llevadas a cabo en la vertiente guipuzcoana de la Sierra de Aralar y su entorno colindante con Navarra (Noval, 1967; Álvarez et al., 1985). A finales de los noventa se detectaron indicios de expansión en esta zona (Aierbe et al., 2001) y aproximadamente en el mismo período se produjeron las primeras observaciones en la zona occidental (hayedo de Altube y montes de Ordunte) (Fernández \& Ruiz de Azua, 1988; Simal \& Herrero, 2003; Lorenzo \& Pérez de Ana, 2003). Las últimas referencias muestran una rápida colonización del territorio vasco, con una clara implantación en la mitad sur y este de Gipuzkoa (Olano et al., 2015) y una ocupación del centro-sureste de Álava, la divisoria de aguas atlántico-mediterránea, los montes vizcaínos de las Encartaciones y otras zonas limítrofes con Burgos (Gainzarain \& Fernández-García, 2012 y 2013).

El presente estudio tiene como objetivo principal analizar la distribución y estimar la abundancia del picamaderos negro en Bizkaia (País Vasco) y zonas limítrofes, un territorio de reciente colonización (Gainzarain \& Fernández-García, 2012 y 2013) sometido en buena parte a una intensa explotación forestal. Así mismo, este trabajo busca aportar información sobre sus preferencias ecológicas y biología de la reproducción que ayude a comprender dicho proceso de colonización y establecer pautas de conservación para una especie considerada amenazada a nivel regional (Bea, 1999).

\section{Métodos}

\section{Área de estudio}

El área de estudio general abarcó las 39 cuadrículas UTM de $100 \mathrm{~km}^{2}$ en las que queda incluida Bizkaia, así como zonas colindantes de Álava, Burgos, Cantabria y Gipuzkoa. Se extiende desde el nivel del mar hasta los 1.481 m de su cumbre más alta, con una altitud media aproximada de $290 \mathrm{~m}$. La vegetación potencial dominante en la mayor parte de su zona costera y colina es el robledal de roble pedunculado Quercus robur L. y el bosque mixto, mientras que a partir de los $600 \mathrm{~m}$ de altitud, el bosque natural dominante sería el hayedo de Fagus sylvatica L. No obstante, la mayor parte de la vegetación natural está muy alterada, principalmente como consecuencia de una histórica intensa actividad agroganadera y una intensa reforestación posterior llevada a cabo esencialmente con especies exóticas (Aseginolaza et al., 1988). En la actualidad, alrededor del 58,6 \% de la superficie de Bizkaia está cubierta por zonas arboladas, la mayor parte cultivos forestales de crecimiento rápido y medio (74,1\%), entre los que destacan las plantaciones de Pino de Monterrey Pinus radiata D.Don y otras coníferas, así como los eucaliptos Eucalyptus spp (Tabla 1). El 23,9 \% de la superficie forestal de Bizkaia es propiedad pública (Montes de Utilidad Pública, Patrimoniales de la Diputa- 


\begin{tabular}{|lccc|}
\hline Sector forestal & \%Bizkaia & \%Territorio & DIF\% \\
\hline Pinus nigra & 2,46 & 3,4 & $0,94^{* *}$ \\
\hline Pinus pinaster & 3,26 & 6,2 & $2,94^{* *}$ \\
\hline Pinus radiata & 49,8 & 58,2 & $8,40^{* *}$ \\
\hline Pseudotsuga menziessi & 1,15 & 1,09 & $-0,06$ \\
\hline Chamaecyparis lawsoniana & 1,28 & 1,05 & $-0,08$ \\
\hline Quercus robur & 1,3 & 1,02 & 1,02 \\
\hline Quercus ilex & 2,22 & 1,89 & $-0,33^{* *}$ \\
\hline Quercus rubra & 1,02 & 2,26 & $1,24^{* *}$ \\
\hline Fagus sylvatica & 4,02 & 6,23 & $2,11^{* *}$ \\
\hline Eucalyptus spp. & 13,3 & 5,66 & $-7,64^{* *}$ \\
\hline Bosque de ribera & 1,25 & 0,15 & $-1,90^{* *}$ \\
\hline Otras caducifolias & 14,20 & 14,93 & 0,73 \\
\hline
\end{tabular}

Tabla 1.- Superficie arbolada ocupada por los principales sectores forestales en Bizkaia (Gobierno Vas$c 0,2018$ ) y en los 36 territorios de picamaderos negro muestreados (** $p<0,001$ ).

Table 1.- Wooded area occupied by the main forest sectors in Bizkaia (Basque Government, 2018) and in the 36 black woodpecker territories sampled (** $p<0.001)$.

ción Foral y de Libre disposición) (Inventario Forestal CAE, 2019). Durante las últimas décadas (1972-2019) la superficie forestal se ha incrementado aproximadamente en un $10 \%$, destacando el aumento en un $612 \%$ de la cubierta por eucaliptos y la reducción en un 25\% de la ocupada por coníferas (ICONA, 1972, Gobierno Vasco, 2019).

\section{Muestreo y análisis de datos}

En primer lugar y con el objetivo de conocer la distribución aproximada de la especie en el área de estudio, se llevó a cabo una recopilación de citas de la especie de los últimos cinco años en las 39 cuadrículas del área de estudio a través de las plataformas OrnithoEus (2020) y eBird (2020).

En segundo lugar, se seleccionaron 28 cuadrículas UTM de $100 \mathrm{~km}^{2}$ (excluyéndose las de predominio marítimo y urbano) con el objetivo de prospectar de forma más uniforme el área de estudio. En 2019, en cada cuadrícula se llevaron a cabo itinerarios de censo a través de las masas forestales añosas potencialmente adecuadas para la especie en los que los participantes anotaron los contactos visuales y sonoros, estandarizándose el esfuerzo de muestreo de forma proporcional a la superficie ocupada por cada una de ellas (Olano et al., 2015). Los itinerarios fueron seleccionados y realizados por agentes forestales en sus zonas habituales de trabajo, y se llevaron a cabo preferentemente durante las primeras horas del día y en ausencia de viento o lluvia 
fuertes, entre febrero y abril, época de máxima detectabilidad de la especie (Bocca et al., 2007), En total se diseñaron 130 itinerarios con una longitud media de $3,6 \mathrm{~km}$ $(0,4-7,8 \mathrm{~km})$ que fueron repetidos un promedio de 2,3 veces y sumaron un total de 300 recorridos $(1075 \mathrm{~km})$.

En tercer lugar, durante la primavera de 2019 se llevó a cabo una búsqueda de nidos, tanto viejos como activos, utilizando para ello la información obtenida en los recorridos y la experiencia previa de cada agente forestal en su zona de trabajo, así como también la información suministrada por terceros relativa a nidificación en años anteriores. Para cada árbol-nido localizado se registró su localización geográfica por el sistema de coordenadas universal transversal de Mercator (UTM), la altitud (m.s.n.m.), la propiedad del suelo (pública/privada), el tipo de formación forestal en el que estaba localizado, la altura a la que se situaba la cavidad, así como la especie de árbol, su estado (vivo/muerto) y diámetro a la altura del pecho (DAP). Cuando fue posible (12 casos en 2019 y 3 en 2018) se llevó a cabo un seguimiento mínimo de los nidos activos con el objetivo de conocer de forma preliminar la fenología local de la reproducción y el número de pollos mayores de 20 días por nido.

En cuarto lugar, se mapearon mediante QGIS las superficies forestales potencialmente utilizables por 36 parejas en círculos con epicentro en sus nidos, asumiendo un tamaño territorial de 250 ha (véase Olano et al., 2015). Como fuente de información se utilizó el Inventario Forestal de 2018 (Gobierno Vasco, 2018). Se evaluó mediante un test $\chi^{2}$ la homogeneidad de las frecuencias del tipo de arbolado más común observada en cada territorio de picamaderos negro $(>1 \%)$ con las frecuencias esperadas calculadas a partir de la extensión de dicho tipo de arbolado en el conjunto de Bizkaia. La asociación de frecuencias entre el estado de los árboles-nido (vivo/muerto) y la categoría general asignada a la especie de árbol (coníferas/otros) se testó también mediante el test de $\chi^{2}$. La comparación entre el porcentaje de nidos en suelo público y el porcentaje de suelo público en Bizkaia se realizó con el test de la z. La normalidad de las variables se testó mediante el test de Kolmogorov-Smirnov y la homogeneidad de sus varianzas mediante el test de Levene. Las variables Diámetro y Altura se transformaron en logaritmos para normalizarlas. Se compararon las medianas de estas tres mismas variables organizadas en tres categorías arbóreas (coníferas / caducifolios / eucaliptos) mediante la prueba no-paramétrica de Kruskal-Wallis (K-W), seguida del test de Dunn de comparación de pares de datos, al no cumplirse la normalidad o la homogeneidad de las varianzas en dos de las tres variables analizadas.

Por último, a partir de las citas recopiladas y los nidos localizados se llevó a cabo una estima de la población. Para ello se consideraron territorios seguros, aquellos sectores forestales en los que en 2019 se detectó un nido o una pareja o bien se contactó nuevamente con la especie al menos en una ocasión en una zona de nidificación conocida. Se consideraron como territorios probables aquellos sectores forestales en los que la especie fue detectada en más de tres ocasiones durante cualquier época del año. 


\section{Resultados}

Se recopilaron 375 citas de la especie en el periodo 2015-2019. Dichas citas se produjeron en la práctica totalidad del área de estudio, aunque fueron menos abundantes en las cuadrículas más cercanas a la costa (fig. 1). Durante la realización de los transectos se escuchó u observó la especie en 41 de los itinerarios diseñados (31,5\%) y en 21 de las cuadrículas (75\%), contabilizándose 0,05 aves/km recorrido.

Se detectaron 50 territorios seguros en base a la localización de nidos activos (29), observación de parejas (7) y la presencia de nidos antiguos combinada con observaciones durante la época reproductora (14). Además, se estimó la existencia de 18 territorios probables. Los territorios se ubicaron en 25 de las 39 cuadrículas del área estudiada (64\%) (fig. 2). El 44,6\% de los territorios seguros se localizaron en el rango altitudinal 400-600 m y el 37,5\% por debajo de los $400 \mathrm{~m}$ (fig. 3).

La cobertura media de la superficie arbolada en torno a los nidos fue del 86,3\% (rango = 53,4-99,2). La extensión de pinares (Pinus pinaster Ait., Pinus nigra Arnold y Pinus radiata), hayedos (Fagus sylvatica) y robledales exóticos (Roble americano, Quercus. rubra L) en los territorios fue significativamente mayor que la esperada, mientras que la de encinares (Quercus ilex L), bosque de ribera y, sobre todo, la de eucaliptales (Eucalyptus spp.) fue menor $(\mathrm{p}<0.001)$ (Tabla 1). El porcentaje de árboles-nido

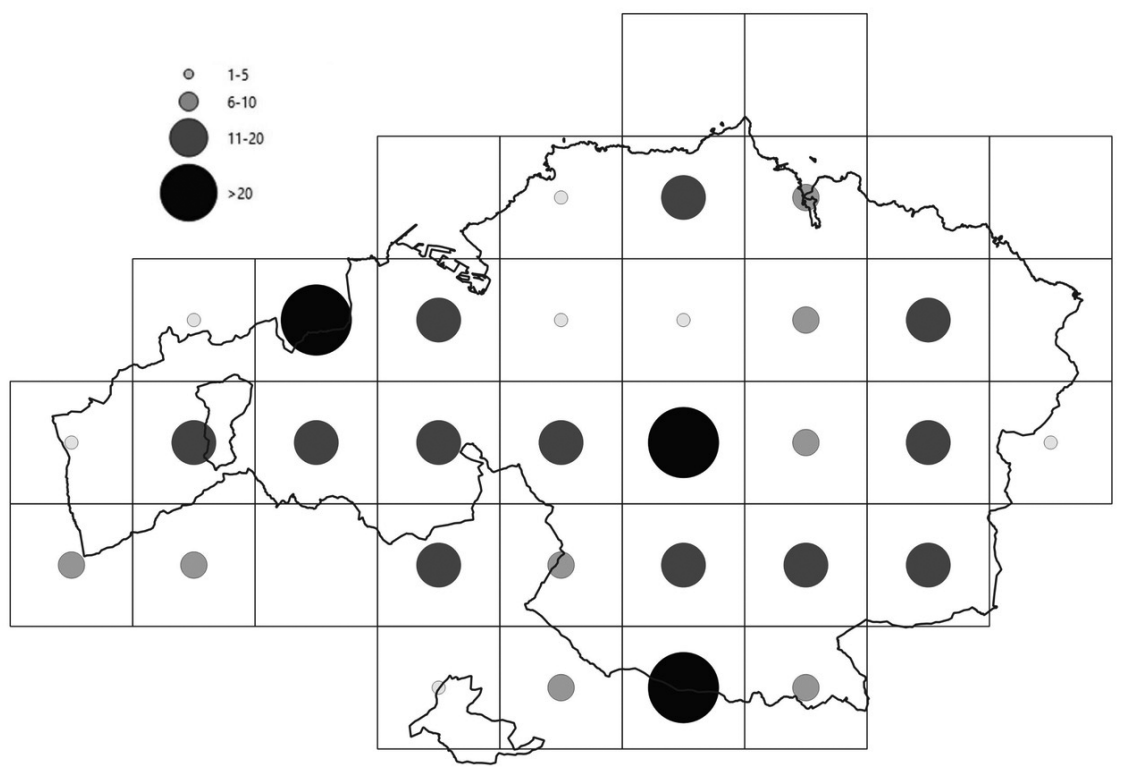

Fig. 1.- Distribución del número de citas de picamaderos negro en Bizkaia y zonas limítrofes en el periodo 2015-2019.

Fig. 1.- Distribution of the number of records of black woodpecker in Bizkaia and neighbouring areas during the period 2015-2019. 


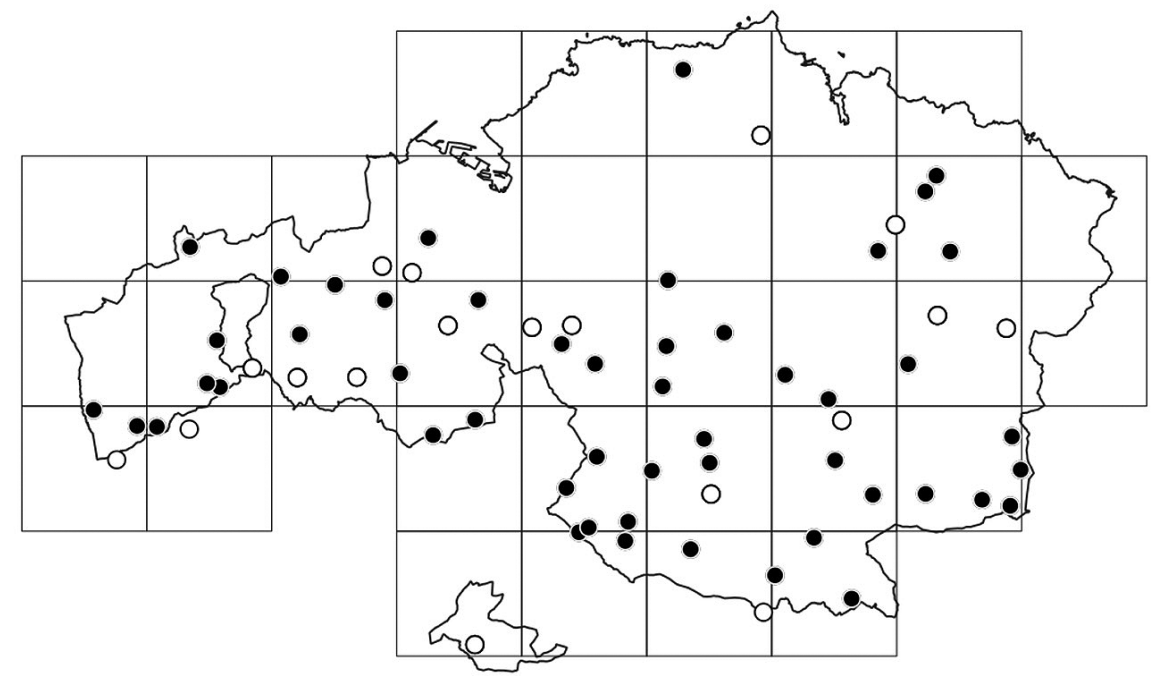

Fig. 2.- Distribución de los territorios seguros (negro) y probables (blanco) de picamaderos negro en Bizkaia y zonas limítrofes.

Fig. 2.- Distribution of the known (black) and probable (white) territories of black woodpecker in Bizkaia and neighbouring areas.

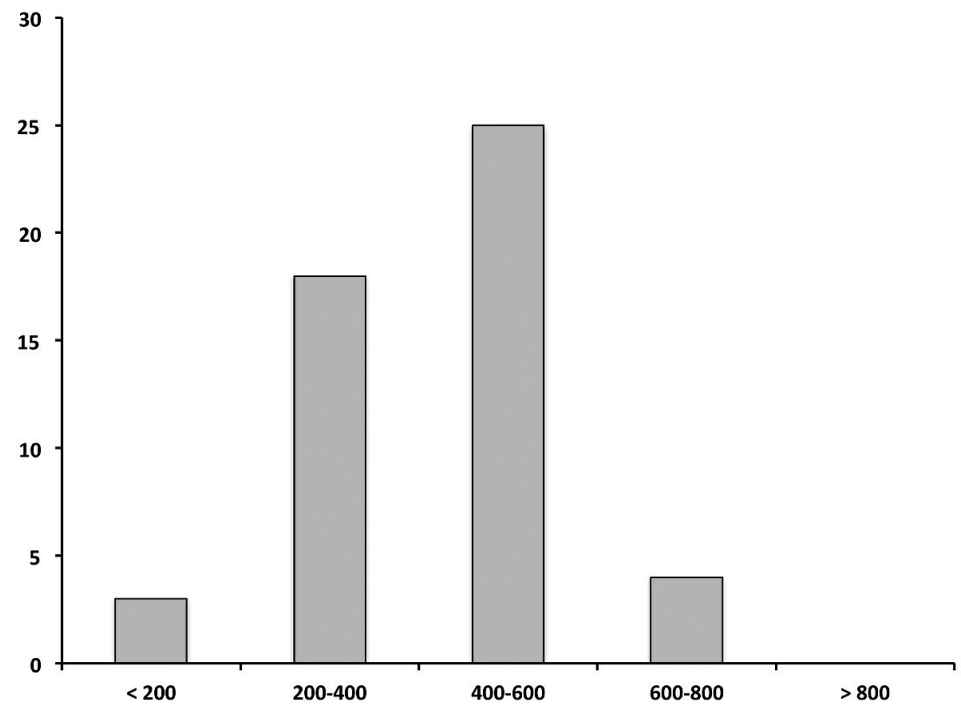

Fig. 3.- Distribución altitudinal (m.s.n.m.) del número de territorios seguros de picamaderos negro en Bizkaia y zonas limítrofes $(n=50)$.

Fig. 3.- Altitudinal distribution (m.a.s.I.) of the number of reliable black woodpecker territories in Bizkaia and neighbouring areas $(n=50)$. 
ubicados en suelo público (38,8\%) fue superior al esperado en relación a la proporción de suelo público forestal en Bizkaia ( $z=-2,09 ; p=0,018)$.

Se midieron las características de 56 árboles-nido (Tabla 2), tanto activos como inactivos, que resultaron pertenecer a seis especies arbóreas: F. sylvatica $(47,2 \%), P$. radiata $(18,2 \%)$, P. pinaster $(12,7 \%)$, Q. rubra (5,4\%), Eucalyptus globulus Labill $(14,5 \%)$ y Eucalyptus nitens $\mathrm{H}$. Deane y Maide (1,8\%). En la mayoría de los casos su identidad específica coincidió con la de la especie arbórea dominante en la parcela, excepto en el caso de los ejemplares de eucaliptos, los cuales invariablemente estuvieron ubicados en plantaciones de coníferas. Cuando utilizaron coníferas como árbol-nido, seleccionaron únicamente ejemplares muertos, que en la mayoría de los casos (94\%) estaban descortezados, mientras que cuando utilizaron otras especies arbóreas la mayoría de los nidos estaban excavados en árboles vivos, siendo dicha asociación significativa $\left(\chi^{2}=46,6 ; p<0,001\right)$ (tabla 2). Los árboles utilizados tuvieron un diámetro medio $( \pm \mathrm{DE})$ de $59,2( \pm 9,9) \mathrm{cm}$ y los nidos estuvieron situados a una altura media $( \pm \mathrm{DE})$ de $9,6( \pm 3,2) m$ (Tabla 2). Se observaron diferencias significativas en el diámetro de los árboles-nido entre coníferas, caducifolias y eucaliptos ( $K-W, p<0,001)$, pero no en la altura en que se localizaban los nidos. Los árboles-nido con menor diámetro (media $\pm D E$ ) fueron las coníferas (test de Dunn, $p<0,01$ ). También se observaron diferencias en la altitud a la que se localizaron los árboles-nido ( $K-\mathrm{W}, \mathrm{p}<0,021)$, con diferencias significativas entre la localización altitudinal de los nidos emplazados en eucaliptos, más baja, y la de los ubicados en caducifolios (test de Dunn, $p=0,029$ ).

El número medio $( \pm \mathrm{DE})$ de pollos registrado en los 15 nidos monitoreados fue 1,9 $( \pm 0,9)$. Dos de ellos fallaron por causas desconocidas y otro resultó depredado por un mamífero carnívoro no identificado. El número medio $( \pm D E)$ de pollos en nidos que criaron exitosamente al menos un pollo fue 2,16 $( \pm 0,5$; rango $=1-3)$. Se observaron relevos de incubación (10 casos) entre el 30 de Marzo y el 16 de Mayo, y cebas (17 casos) entre el 28 de Abril y el 4 de Junio. Se estima que los pollos abandonaron los nidos entre el 18 de mayo y el 17 de junio (7 casos).

\begin{tabular}{|lcc|}
\hline Especie (vivo/muerto) & Diámetro $( \pm \mathrm{DE})($ Rango) & Altura $( \pm \mathrm{DE})($ Rango $)$ \\
\hline Fagus sylvatica $(26 / 1)$ & $59,8 \pm 7,1(39-90)$ & $8,6 \pm 2,6(6-15)$ \\
\hline Pinus radiata $(0 / 10)$ & $47,1 \pm 4,1(43-58,2)$ & $8,0 \pm 3,6(5,2-15)$ \\
\hline Eucalyptus spp.(8/1) & $60,5 \pm 10,7(43-78)$ & $10,8 \pm 3,6(6,5-18,4)$ \\
\hline Pinus pinaster (0/7) & $42,2 \pm 8,7(36-60)$ & $8,2 \pm 0,8(7,5-9,6)$ \\
\hline Quercus rubra (3/0) & $54,3 \pm 7,3(45-63)$ & $12,5 \pm 3,5(10-17,5)$ \\
\hline TOTAL (37/19) & $55,5 \pm 1,0(36-90)$ & $9,1 \pm 3,3(5,2-18,4)$ \\
\hline
\end{tabular}

Tabla 2.- Especie de árbol, su estado, diámetro a la altura del pecho $(\mathrm{cm})$ y altura a la que se situaba el nido $(\mathrm{m})$ para 56 árboles-nido de picamaderos negro en Bizkaia.

Table 2.- Tree species, its condition, diameter at breast height $(\mathrm{cm})$, and height at which the nest was located $(\mathrm{m})$ for 56 black woodpecker nest-trees in Bizkaia. 


\section{Discusión}

En los últimos años la distribución del picamaderos negro se ha ampliado a buena parte del territorio de Bizkaia, lo cual muestra que se está fortaleciendo el proceso de fusión de las poblaciones ibéricas en el País Vasco (Gainzarain \& Fernández-García, 2013 y 2015). Dichas poblaciones han estado históricamente asociadas a bosques maduros del piso montano y subalpino, sobre todo hayedos $y$, en menor medida, abetales, pinares y robledales, situados por lo general por encima de los 600-800 m de altitud (Muntaner et al., 1983; Simal \& Herrero, 2003; Martínez-Vidal, 2004; SánchezCorominas et al., 2006-2009). Aunque la expansión en el País Vasco ha estado inicialmente ligada al hayedo (Gainzarain \& Fernández-García, 2013 y 2015), el bosque característico de su piso montano, este estudio revela que la especie está colonizando también el piso colino hasta el mismo nivel del mar, fuera ya por tanto de la zona de distribución natural del haya.

Se ha localizado medio centenar de territorio seguros, cifra que puede considerarse como el tamaño mínimo de la población estudiada. Esta cifra dobla la población estimada recientemente en otras zonas del País Vasco (Gainzarain \& Fernández-García, 2012 y 2015), aunque la diferencia debe tomarse con cautela ya que puede estar causada por un mayor esfuerzo y cobertura del muestreo. No obstante, también podría ser debido a que, al tratarse de un proceso colonizador reciente, los muestreos llevados a cabo en etapas iniciales únicamente detectan una parte de las parejas que finalmente ocupan una determinada región. En este sentido, futuros estudios ayudarán a comprender con mayor precisión el alcance y la evolución de esta colonización en la región.

La asociación positiva de los árboles-nido de picamaderos negro con un entorno de hayedos, robledales de roble americano y coníferas (principalmente, pinos) sugiere que estos ambientes forestales juegan un importante papel en la colonización del área de estudio. Algunos autores (Olano et al., 2015) han sugerido que la potencialidad de las plantaciones de pino de Monterrey está limitada por su escasez en recursos tróficos y en árboles adecuados para la nidificación, mientras que otros autores (Robles et al., 2020) no descartan que estas plantaciones tengan relevancia en la búsqueda de alimento. Aun sin desmentir sus posibles limitaciones, en este estudio se ha observado que los pinos de Monterrey suponen un porcentaje importante de los territorios de cría del picamaderos negro y que además los utiliza como árboles-nido. Teniendo en cuenta su extensa distribución en la región (Tabla 1) y su fuerte implantación desde el nivel del mar hasta el piso montano, las plantaciones de pino de Monterrey estarían siendo, por tanto, cruciales en el proceso colonizador del área estudiada.

La distribución del picamaderos negro está en parte condicionada por la disponibilidad de árboles adecuados para la nidificación, habitualmente ejemplares altos, sin ramas bajas y con un diámetro mínimo de 26 cm dap (Roldstad, 2000; Camprodon et al., 
2007; Pirovano \& Zecca, 2014). Sin embargo, se muestra muy generalista a la hora de seleccionar la especie arbórea, habiéndose descrito al menos 50 especies a lo largo de su área de distribución mundial (Gorman, 2011).

El haya, en su área de distribución natural, es una de las especies arbóreas más utilizadas como árbol-nido de los bosques templados europeos (Fernández \& Azkona, 1996; Bocca et al., 2007; Kosinski et al., 2010; Simal \& Herrero, 2003; Pirovano \& Zecca, 2014; Saporetti et al., 2016) y también en los bosques recientemente colonizados del País Vasco (Gainzarain \& Fernández-García, 2015; Robles et al., 2020), confirmándose en el presente trabajo su importancia a escala regional. La selección positiva del haya como árbol-nido se atribuye al gran tamaño y porte rectilíneo que alcanza, a su carencia de ramas bajas y a su corteza lisa, factores que dificultarían el acceso de los depredadores terrestres a los nidos (Gorman, 2011). El haya comparte estas características con el roble americano y los eucaliptos, árboles que también utiliza el picamaderos negro para excavar sus nidos en el área de estudio. El roble americano había sido citado como árbol-nido, tanto en el País Vasco como en otras localidades europeas (Gainzarain \& Fernández-García, 2015; Saporetti et al., 2016), mientras que la ocupación de eucaliptos no había sido reportada hasta la fecha en la literatura científica. En el área de estudio, el picamaderos negro utiliza también el pino marítimo Pinus pinaster y pino de Monterrey, ambas especies tampoco citadas anteriormente como árboles nido y que se cultivan en el piso colino de la región. Se trata, por tanto, de un claro indicio de que la expansión no sólo se está produciendo en los pisos montanos y subalpinos, sino que afecta también a zonas más atemperadas, en las que desde hace décadas se cultivan estas dos especies arbóreas exóticas como alternativa a la histórica deforestación y retracción posterior de las actividades agropecuarias (Tellería \& Galarza, 1990). Todos los árboles-nido localizados en ejemplares del género Pinus estaban muertos, lo que sugiere una selección negativa de los pinos vivos, consecuencia probable de su alto contenido en resinas (Rolstad et al., 2000; Pirovano \& Zecca, 2014). Seleccionan además pinos descortezados con el probable objetivo de reducir la depredación, del mismo modo que tienden a seleccionar árboles vivos de corteza lisa, aunque se desconoce si los descortezan ellos mismos o si eligen ejemplares ya descortezados. La positiva selección de los montes de propiedad pública sugiere una explotación silvícola más compatible con la especie debido quizás en parte a la composición específica de estos bosques plantados o naturales, así como a la explotación en turnos de corta más largos.

El éxito reproductor de la especie fue alto (80\%), similar al obtenido en zonas montanas cercanas del País Vasco (Gainzarain \& Fernández-García, 2015; Robles et al., 2020) y a la media europea (Passinelli, 2006). El número medio de pollos volados por nido $(2,1)$ fue, sin embargo, menor que la media observada a nivel europeo $(3,3)$ (Passinelli, 2006). La baja tasa de pollos registrada en éste y otros estudios llevados a cabo en el País Vasco podría ser un indicador de baja disponibilidad de alimento 
(Gainzarain \& Fernández-García, 2015; Robles et al., 2020), aunque también se ha sugerido que podría estar causada por una falta de experiencia reproductora en una población que a priori se supone formada por ejemplares jóvenes, recientemente establecidos (Gainzarain \& Fernández-García, 2015). No obstante, la información disponible sobre el éxito reproductor en el área de estudio debe tomarse con cautela al provenir de un pequeño tamaño de muestra tomado en un corto periodo de tiempo.

\section{Consideraciones conservacionistas}

La histórica deforestación y la fragmentación de buena parte de los bosques naturales significó la rarefacción o extinción local de las aves forestales más sensibles en amplios sectores del norte de la península Ibérica (Tellería \& Galarza, 1990). Posteriormente, la intensa repoblación forestal llevada a cabo sobre todo a partir de mediados del siglo pasado (Michel, 2006; Uriarte, 2010), aumentó de forma extraordinaria la superficie arbolada, en especial la cubierta con coníferas de crecimiento rápido, lo que favoreció la colonización de la avifauna forestal (Carrascal \& Tellería, 1990; Tellería \& Galarza, 1990). No obstante, este efecto restaurador ha estado muy limitado por la rápida dinámica de plantación, cuidado y explotación de la mayoría de estos cultivos forestales, que dificulta una configuración estable de arbolado añoso y obstaculiza el asentamiento de las especies típicas de los estadios maduros (Tellería, 1992).

La intensa reforestación con coníferas llevada a cabo durante el siglo pasado, así como la maduración del bosque natural caducifolio y una explotación silvícola menos intensiva se han barajado como responsables de la expansión que ha experimentado el picamaderos negro a escala europea (Mikusinski, 1995; Gorman, 2004 y 2011; Camprodon et al., 2007; Gil-Tena et al., 2010). También en el área de estudio la expansión ha de estar relacionada, al menos parcialmente, con la maduración de las masas forestales, dado que su reforestación intensiva se inició hace apenas siete décadas (Michel, 2006; Uriarte, 2010). Además, el retraso en los turnos de corta o a la reducción de las talas por disminución de la demanda de madera habría mejorado la biodiversidad silvícola y la presencia de arbolado maduro (Gainzarain \& Fernández-García, 2013). A este proceso se habrían añadido la sucesión de eventos extremos de las últimas décadas (incendios, plagas forestales y meteoros), como consecuencia de los cuales el $40 \%$ de los árboles del País Vasco estaría actualmente dañado (Cantero, 2019). Ello habría aumentado la abundancia de coníferas muertas susceptibles de ser utilizadas para la nidificación y como fuente de alimento, tanto por el picamaderos negro como también por otras especies. Así, por ejemplo, se ha observado cómo el pico picapinos Dendrocopus major (Linnaeus, 1758) y el trepador azul Sitta europea Linnaeus, 1758 han colonizado profusamente las repoblaciones costeras de pino de Monterrey, un hábitat del área de estudio en donde las dos especies estaban ausentes o eran raras a finales del siglo pasado (Noval, 1967; Alvarez et al., 1985; Fernández \& Galarza, 1986; Carrascal \& Tellería, 1990; Tellería \& Galarza, 1990; Aierbe et al., 2001; De la Hera et al., 2013). En esta última década, la infección y agresiva propagación 
de hongos defoliadores, sobre todo Lecanosticta acicola (banda marrón), está produciendo importantes daños que afectan en particular a las plantaciones de pino de Monterrey (Cantero, 2019). Este proceso estaría incrementando, al menos temporalmente, la disponibilidad de alimento y, como consecuencia, la abundancia de pícidos, efecto bien documentado en Norte América (Murphy \& Lehnhausen, 1998; Edworthy et al., 2011; Saab et al., 2019). Sin embargo, las repoblaciones de pinos afectadas están siendo sustituidas mayoritariamente por eucaliptos, acelerando de este modo una práctica forestal observada ya en las últimas décadas en el área de estudio.

El análisis de la cubierta forestal en torno a los árboles-nido sugiere que la especie no selecciona el eucaliptal como entorno para la reproducción. Este cultivo muestra grandes limitaciones tróficas y estructurales para la avifauna y es escasamente ocupado por los pájaros carpinteros (Pina, 1989; Tellería \& Galarza, 1990 y 1991; Santos \& Álvarez, 1990; De la Hera et al., 2013). Además, los eucaliptales son masas forestales caracterizadas por una alta densidad arbórea y elevada cobertura arbustiva (Tellería \& Galarza, 1990 y 1991), que podría limitar las posibilidades de alimentación del picamaderos negro, ya que la especie evita los bosques provistos de matorral denso (Bocca et al., 2007). Además, debido a que su madera se utiliza esencialmente para pasta de papel, la mayoría de estos cultivos se explota a matarrasa en turnos de corta de 1014 años (Veiras \& Soto, 2011) por lo que los árboles no alcanzan habitualmente el diámetro mínimo necesario para cobijar nidos de picamaderos negro, a pesar de la idoneidad de la especie. De hecho, los nidos registrados se observaron únicamente en eucaliptos viejos (30-65 años) de grandes dimensiones (Tabla 2) que crecen dispersos en plantaciones de pinos.

Desde el punto de vista de la gestión, la mayoría de los autores coinciden en que sería deseable una política forestal que favoreciera la conservación y la madurez de todos los tipos de vegetación que habita la especie y también la preservación de sus árboles-nido durante las labores silvícolas (Rolstad et al., 2000; Cárcamo, 2006; Camprodon et al., 2007; Pirovano \& Zecca, 2014). En el área de estudio, una región fuertemente parcelada y sometida a una intensa explotación forestal, el picamaderos negro podría mantener una población estable compensando la escasez de bosques naturales maduros y extensos con la utilización de múltiples parcelas de otros tipos de arbolado añoso integrados en territorios más amplios (Tjernberg et al., 1993; Schmiegelow \& Monkkonen, 2002). Sin embargo, se hace urgente limitar la sustitución de las coníferas por eucaliptales, cultivos forestales que no solamente suponen un importante freno para la colonización del picamaderos negro, sino que además están causando un grave deterioro en la abundancia y diversidad de la avifauna regional (Tellería \& Galarza, 1990 y 1991; De la Hera et al., 2013). En general, se hace imprescindible incorporar pautas de gestión forestal específicas en las estrategias de conservación de la biodiversidad regional (Diputación Foral de Bizkaia, 2015) que favorezcan al picamaderos negro y a otras aves forestales, además de incluir la preservación 
de esta especie como uno de los objetivos prioritarios en los planes de gestión de los montes de propiedad pública.

\section{Agradecimientos}

Nuestro agradecimiento a Kepa Aldama, Juan Carlos Andrés, Mitxel Andreu, Aitor Arizmendiarrieta, Eduardo Aurrekoetxea, Gorka Ezenarro, Gorka Hurtado, Iñigo Ibaibarriaga, Eusebio Jaio, Julio Rodríguez e Iñigo Zuberogoitia, por facilitar información sobre observaciones y nidos. José Mari Fernández-García (HAZI Fundazioa) contribuyó en aspectos relacionados con el diseño inicial. Ángel Zumalakarregi (DFB/BFA) facilitó información relativa a la edad de los árboles-nido. Pilar Rodriguez (UPV/EHU) contribuyó con sus comentarios a mejorar una primera versión del trabajo. David Álvarez (Editor asociado) y Susana Cárcamo realizaron una última revisión del manuscrito presentado para su publicación.

\section{Bibliografía}

Álvarez, J., Bea, A., Faus, J.M., Castién, E., Mendiola, I., 1985. Atlas de los vertebrados continentales de Álava, Vizcaya y Guipúzcoa (excepto Chiroptera). Gobierno Vasco, Bilbao.

Aierbe, T., Olano, M., Vázquez, J., 2001. Atlas de las aves nidificantes de Gipuzkoa. Munibe, Cienc. nat. 52, 5-138.

Aseginolaza, C., Gómez, D., Lizaur, X., Monserrat, G., Morante, G., Salaverria, M.R., Uribe-Echebarria, P., 1988. Vegetación de la Comunidad Autónoma del País Vasco. Gobierno Vasco, Bilbao.

Bagne, K.E., Purcell, K.L., Rotenberry, J.T., 2008. Prescribed fire, snag population dynamics, and avian nest site selection. For. Ecol. Manag. 255, 99-105.

Bea, A., 1999. Vertebrados amenazados del País Vasco. Servicio Central de Publicaciones del Gobierno Vasco, Vitoria-Gasteiz.

Bocca, M., Carisio, L., Rolando, A., 2007. Habitat use, home ranges and census technoques in the Black Woodpecker Dryocopus martius in the Alps. Ardea 95, 17-29.

Cantero, A., 2019. Proyecto LIFE Healthy Forest. Informe final. Fundación Hazi. Arkaute. Disponible en: https://www.hazi.eus/images/documentos/forestal/hforest.pdf.

Cárcamo, S., 2006. Evolución de las poblaciones de pito negro (Dryocopus martius) y pico dorsiblanco (Dendrocopus leucotos liffordi) en los montes de Quinto Real (Navarra) y su relación con la gestión forestal. Pirineos 161, 133-150.

Carrascal, L.M., Tellería, J.L., 1990. Impacto de las repoblaciones de Pinus radiata sobre la avifauna forestal del norte de España. Ardeola 37(2), 247-266. 
Camprodon, J., Campión, D., Martínez-Vidal, R., Onrubia, A., Robles, H., Romero, J.L., Senosiain, A., 2007. Estatus, selección del hábitat y conservación de los pícidos ibéricos. En: Camprodon, J., Plana, E. (Eds.), Conservación de la biodiversidad y gestión forestal, 391-434. Universidad de Barcelona, Barcelona.

De la Hera, I., Arizaga, J., Galarza, A., 2013. Exotic tree plantations and avian conservation in northern Iberia: a view from a nest-box monitoring study. Anim. Biodivers. Conserv. 36(2), 153-162.

Del Hoyo, J., Elliot, A., Sargatal, J., 2002. Handbook of the Birds of the World. Vol. 7. Lynx Edicions, Barcelona.

De Rosa, D., Andriuzzi, W.S., Febbraro, M.D., 2016. Breeding hábitat selection of the Black Woodpecker Dryocopus martius L. In: Mediterranean forests, 63-69. Avocetta 40.

Diputación Foral de Bizkaia, 2015. Estrategia para la protección, mejora y gestión de la biodiversidad en Bizkaia. Disponible en: www.bizkaia.eus.

eBird 2020. eBird Basic Dataset. Cornell Lab. Of Ornithology, Ithaca, New York. Available from: https://ebird.org/spain/science.

Edworthy, A.B., Drever, M.C., Martin, K., 2011. Woodpeckers increase in abundance but maintain fecundity in response to an outbreak of mountain pine bark beetles. For. Ecol. Manag. 261, 203-210.

Fernández, C., Azkona, P., 1996. Influence of forest structure on the density and distribution of the White-backed Woodpecker Dendrocopus leucotos and Black Woodpecker Dryocopus martius in Quinto Real (Spanish western Pyrenees). Bird Study 43, 305-313.

Fernández, A., Galarza, A., 1986. Estructura y estacionalidad de las comunidades de aves en distintos medios del tramo costero del País Vasco. Bol. de la Est. Central Ecol. 29, 5966.

Gainzarain, J.A., Fernández-García, J.M., 2012. Distribución y población del picamaderos negro Dryocopus martius en Álava. Año 2011. Informe inédito.

Gainzarain, J.A., Fernández-García, J.M., 2013. Black woodpecker Dryocopus martius (L., 1758) recent range expansion leads to the coalescence of the two former distribution areas in northern Spain. Munibe, Cienc. nat. 61, 103-115.

Gainzarain, J.A., Fernández-García, J.M., 2015. Black woodpecker Dryocopus martius (L., 1758) distribution, abundance, habitat use and breeding performance in a recently colonized region in SW Europe. Munibe, Cienc. nat 63, 49-71.

Garmendia, A., Cárcamo, S., Schwendtner, O., 2006. Forest management considerations for conservation of Black woodpecker Dryocopus martius and White-backed Woodpecker Dendrocopus leucotos populations in Quinto Real (Spanish Western Pyrenees). Biodivers. Conserv. 15, 1399-1415.

Gil-Tena, A., Brotons, L., Saura, S., 2010. Effects of forest landscape change and management on the range expansion of forest bird species in the Mediterranean region. For. Ecol. Manag. 259, 1338-1346. 
Gobierno Vasco, 2018. Inventario Forestal CAE 2018. Disponible en: www.euskadi.eus. Gobierno Vasco, 2019. Mapa Forestal CAE 2019. Disponible en: www.euskadi.eus. Gorman, G., 2004. Woodpeckers of Europe. A study of the European picidae. Bruce Coleman Ed. Berkshire, U.K.

Gorman, G., 2011. The Black Woodpecker. A monograph on Dryocopus martius. Lynx Edicions, Barcelona.

ICONA, 1972. Inventario Forestal Nacional. Subdirección General de Protección de la Naturaleza. Madrid.

Inventario Forestal CAE, 2019. Gobierno Vasco. Disponible en: www.euskadi.eus.

Johnson, K., Nilsson, S.G., Tjernberg, M., 1990. The Black woodpecker; a key-species in european forests. In: Carlsson, A., Aulen, G. (Eds.), Conservation and management of woodpecker populations, 99-102. Swedish University of Agriculture Sciences, Department of Wildlife Ecology, Report 17. Uppsala.

Kosinski, Z., Bilinska, E., Derezinski, J., Kempa, M., 2010. The Black Woodpecker Dryocopus martius and the beech Fagus sylvatica as keystone species for the Stock Dove Columba palumbus in western Poland. Ornis Polonica 51, 1-13.

Lorenzo, J.C., Pérez de Ana, J.M., 2003. Picamaderos negro, Dryocopus martius. Noticiario Ornitológico. Ardeola 50(1), 165.

Martínez-Vidal, R., 2004. Pico negre Dryocopus martius. En: Estrada, J., Pedrocchi, V., Brotons, L. (eds.), 2004. Atles dels ocells nidificant de Catalunya 1999-2002. Institut Catalá d'Ornitologia. Lynx Edicions, Barcelona.

Michel, M., 2006. El Pino radiata en la historia forestal vasca. Análisis de un proceso de forestación intensivo. Munibe, Supl. 23.

Mikusinski, G., 1995. Population trends in black woodpecker in relation to changes and characteristics of European forests. Ecography 18, 363-369.

Mikusinski, G., Gromadzki, M. and Chylarecki, P., 2001. Woodpeckers as indicators of forest bird diversity. Conserv. Biol. 15, 208-217.

Muntaner, J., Ferrer, X., Martínez-Vilalta, A., 1983. Atlas del ocells nidificants de Catalunya i Andorra. Ketres Editora, Barcelona.

Murphy, E.C., Lehnhausen, W.A., 1998. Density and foraging ecology of woodpeckers following a stand-replacement fire. J. Wildl. Manag 62, 1359-1372.

Noval, A., 1967. Estudio de la avifauna de Guipúzcoa. Munibe 19(1-2), 5-78.

Olano, M., Aierbe, T., Beñaran, H., Hurtado, R., Ugarte, J., Urruzola, A., Vázquez, J., Ansorregi, F., Galdos, A., Gracianteparaluceta, A., Fernández-García, J.M., 2015. Black woodpecker Dryocopus martius (L., 1758) distribution, abundance, habitat use and breeding performance in a recently colonized region in SW Europe. Munibe, Cienc. nat 63, 49-71. 
OrnithoEus. 2020, Sistema de Información de la Naturaleza de Euskadi, www.euskadi.eus. Sociedad de Ciencias Aranzadi - Portal Ornitho.eus.

Passinelli, G., 2006. Population biology of European woodpecker species: a review. Ann. Zool. Fennici 43, 96-111.

Pina, J.P., 1989. Breeding bird assemblages in eucalyptus plantations in Portugal. Ann. Zool. Fennici 26, 287-290.

Pirovano, A.R., Zecca, G., 2014. Black Woodpecker Dryocopus martius habitat selection in the Italian Alps: implications for conservation in Natura 2000 network. Bird Conserv. Int. 24, 299-315.

Robles, H., Ciudad, C., Porro, Z., 2020. Selección del Picamaderos negro en el Parque Natural de Aiako Harria. Informe técnico-científico. Habios. Interreg Poctefa.

Rolstad, J., Majewski, P., Rolstad, E., 1998. Black Woodpecker use of habitats and feeding substrates in a managed Scandinavian forest. J. Wildl. Manag 62, 11-23.

Rolstad, J., Rolstad, E., Saeteren, O., 2000. Black Woodpecker nest sites: characteristics, selection, and reproductive success. J. Wildl. Manag 64(4), 1053-1066.

Saab, V.A., Latif, Q.S., Dresser, M.A., Dudley, J.G., 2019. Woodpecker nest survival, density, and a pine beetle outbreak. J. Wildl. Manag. 83, 1387-1400.

Sánchez-Corominas, T., González-Quirós, P., Vázquez, V.M., 2006-2009. El Pico mediano (Dendrocopus medius), el Pico menor (Dendrocopus minor) y el Picamaderos negro (Dryocopus martius), (Picidae, Aves), en el Principado de Asturias (España). Bol. Cienc. Nat. R.I.D.E.A. 50, 281-302.

Santos, T., Álvarez, G., 1990. Efectos de las repoblaciones de aves forestales en un maquis mediterráneo. Ardeola 37(2), 319-324.

Saporetti, F., Colaone, S., Guenzani, W., Zarbo, T., 2016. Nest-site characteristics and breeding biology of the Black Woodpecker in north-western Lombardy, Italy. Revista Italiana de Ornitologia 86(1), 39-48.

Schmiegelow, F.A., Monkkonen, M., 2002. Habitat loss and fragmentation in dynamic landscapes: avian perspectives from the boreal forest. Ecol. Appl. 12(2), 375-389.

Simal, R., Herrero, Á., 2003. Pito negro, Dryocopus martius. En: Martí, R., Moral, J.C. del (Eds.), Atlas de las Aves Reproductoras de España, 354-355. Dirección General de Conservación de la Naturaleza-Sociedad Española de Ornitología.

Tellería, J.L., 1992. Gestión forestal y conservación de aves en España peninsular. Ardeola 39(2), 99-114.

Tellería, J.L., Galarza, A., 1990. Avifauna y paisaje en el norte de España: efecto de las repoblaciones con árboles exóticos. Ardeola 37(2), 229-245.

Tellería, J.L., Galarza, A., 1991. Avifauna invernante en un eucaliptal del norte de España. Ardeola 38(2), 239-247. 
Tjernberg, M., Johnsson, K., Nilsson, S. G., 1993. Density variation and breeding success of the Black Woodpecker in relation to forest fragmentation. Ornis Fenn. 70, 155-162.

Uriarte, R., 2010. Repoblaciones, paisaje forestal y desarrollo industrial en el País Vasco (1940-1975). Historia Agraria 51, 109-142.

Veiras, X., Soto, M.A., 2011. La conflictividad de las plantaciones de eucalipto en España (y Portugal). Análisis y propuestas para solucionar la conflictividad ambiental y social de las plantaciones de eucalipto en la península Ibérica. Greenpeace.

\section{वै)}

Fecha de recepcion/Date of reception: 27/04/2020

Fecha de aceptacion/Date of acceptance: 28/08/2020

Editor Asociado/Associate editor: David Álvarez 